\title{
Local ideal magnetohydrodynamic stability of a quasihelically symmetric stellarator
}

\author{
J. N. Talmadge ${ }^{a}$ \\ Torsatron/Stellarator Laboratory, University of Wisconsin-Madison, Madison, Wisconsin 53706-1691 \\ W. A. Cooper \\ Centre de Recherches en Physique des Plasmas, Association Euratom-Confédération Suisse, \\ Ecole Polytechnique Fédérale de Lausanne, CRPP-PPB, CH-1015 Lausanne, Switzerland
}

(Received 28 March 1996; accepted 19 June 1996)

\begin{abstract}
The ideal magnetohydrodynamic stability with respect to localized ballooning modes and the Mercier criterion of a quasihelically symmetric stellarator configuration is investigated. Configurations with an enhanced magnetic mirror and a deeper magnetic well that can be achieved by energizing a set of auxiliary coils have also been analyzed. In all cases, the Mercier criterion imposes a slightly stricter $\beta$ limit than the ballooning modes. Using relatively broad pressure profiles, the Mercier criterion yields $\beta=0.4 \%$ for the standard configuration, $\beta=0.3 \%$ for the high mirror configuration and $\beta=1.3 \%$ for the deep well configuration. The comparable ballooning limits are $\beta=0.7 \%, \beta=0.6 \%$, and $\beta=1.66 \%$ for the standard, high mirror, and deep well configurations, respectively. With a more peaked pressure profile, ballooning instabilities near the center of the plasma and Mercier modes near the edge of the plasma limit the performance of the standard configuration at $\beta \simeq 0.8 \%$. (C) 1996 American Institute of Physics.
\end{abstract}

[S1070-664X(96)00210-8]

\section{INTRODUCTION}

Ideal magnetohydrodynamic (MHD) stability theory has become a very useful tool to predict the potential operating windows of magnetically confined plasma configurations. With the development of three-dimensional (3D) equilibrium codes such as VMEC, ${ }^{1}$ it is now possible to extend the analysis from axisymmetric and averaged stellarator configurations to fully 3D devices. In particular, local ideal MHD stability investigations as described by the Mercier criterion ${ }^{2}$ and the ballooning mode equation ${ }^{3-5}$ have been carried out extensively for low shear stellarators ${ }^{6-8}$ and high shear torsatrons ${ }^{9-12}$ based on computed equilibria with imposed nested magnetic flux surfaces.

In this paper, we examine the Mercier and ballooning stability of a specific type of low shear stellarator in which the magnetic field structure displays conditions of quasihelical symmetry. ${ }^{13-16}$ In particular, we concentrate our investigations on configurations that model the Helically Symmetric eXperiment (HSX) ${ }^{17}$ at the University of WisconsinMadison. We analyze the local ideal MHD properties of the standard quasihelical configuration and for comparison purposes we examine two other configurations where the quasihelical symmetry is degraded but offer other interesting physical properties.

The paper is arranged as follows. In Sec. II, we describe the HSX configurations we investigate and how we determine the plasma boundary. In Sec. III, we present the basic characteristics and the profiles of the equilibria that model the different HSX configurations. Section IV examines the 3D ideal Mercier and ballooning stability of the HSX model equilibria, followed by conclusions.

a)Electronic mail: u1754@c.nersc.gov

\section{DESCRIPTION OF THE HSX CONFIGURATIONS}

The HSX device is a helical magnetic axis stellarator configuration with 4 field periods, a magnetic field of $1 \mathrm{~T}$, a major radius of $1.2 \mathrm{~m}$, an average minor radius of $0.15 \mathrm{~m}$, and a helical excursion of the magnetic axis of more than 0.2 $\mathrm{m}$. The device consists of 12 modular coils in each period that have been optimized to realize a configuration with quasihelical symmetry. This implies that a Fourier decomposition of the magnetic field strength in Boozer magnetic coordinates ${ }^{18}$ produces one dominant (helical) component in addition to the mean component. We refer to this as the standard configuration and it has a magnetic well of $0.4 \%$. The predicted neoclassical transport losses in the low collisionality regime for a quasihelical stellarator are comparable to those of an axisymmetric tokamak and are much smaller than those of a conventional stellarator. There are an additional 12 auxiliary modular coils in each field period. A current in these coils that is $10 \%$ of that in the main coils increases the magnetic well to $1.5 \%$ at the expense of deteriorating the quasisymmetry properties that characterize the standard configuration. We refer to this as the deep well configuration. Furthermore, reversing the direction of the current in the auxiliary coils at the ends of each field period with respect to the coils in the center excites a toroidal mirror component $(n=1, m=0)$ in the Boozer spectrum. The effect of this component is analogous to that of the toroidal curvature ( $n=0, m=1$ component) in a conventional stellarator and allows the neoclassical transport in HSX to vary from that comparable to a tokamak to that comparable to a conventional stellarator. ${ }^{19}$ The Boozer toroidal mode number (per field period) is $n$ and the poloidal mode number is $m$. This change in the transport can be produced with only small changes in the plasma volume, rotational transform and well depth. In this paper, we will show that the stability limits for 


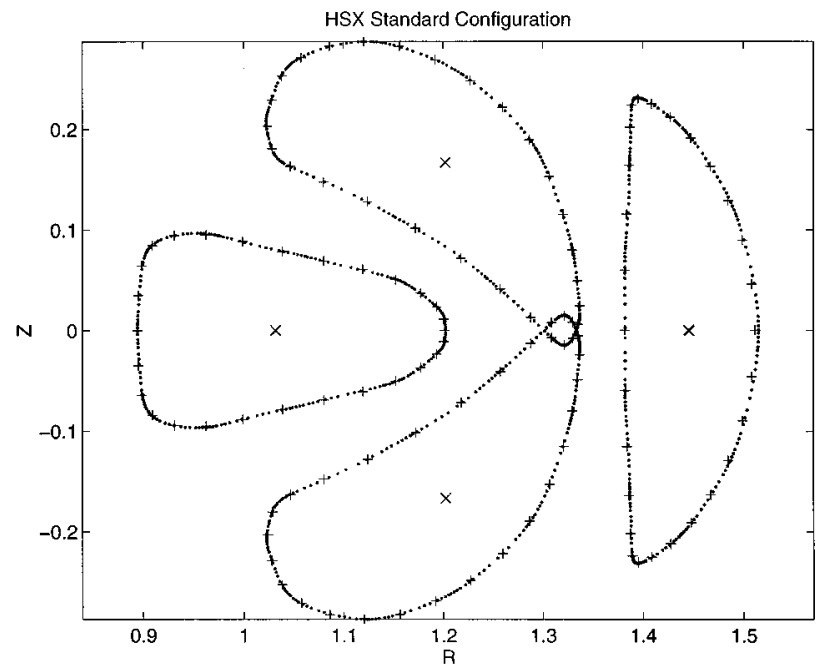

FIG. 1. Poincaré plot obtained from tracing a vacuum magnetic field line started at $R=1.515 \mathrm{~m}, Z=0$ on the elongated up-down symmetric toroidal cross section on 4 planes within one field period (dots) for the standard HSX configuration. This surface is Fourier analyzed and then reconstructed in real space to yield the + symbols. The $\times$ identifies the location of the magnetic axis in the vacuum.

the two configurations are comparable as well. We refer to this as the high mirror configuration and it has a $0.3 \%$ well.

To determine the outermost closed magnetic flux surface, we trace vacuum magnetic field lines starting at different values of $R$, the distance from the major axis, from the midplane of the toroidal cross section at the beginning of a field period. For the standard configuration, we find that tracing field lines from $R=1.515 \mathrm{~m}$ yields the outermost flux surface. In Fig. 1, the dots constitute a Poincare plot that identifies the crossing of the field line we are tracing through 4 different toroidal cross sections within one field period. The position of the magnetic axis (in vacuum) is identified by the $X$ symbol on each cross section. The outer boundary for the high mirror configuration is shown in Fig. 2 by starting the trace at $R=1.495 \mathrm{~m}$. We also show in this figure a Poincare plot of a field line that is started $5 \mathrm{~mm}$ further out. In this case, the field line shows a stochastic character without defining a flux surface quite so clearly. The field line that determines the outermost surface in the deep well configuration is traced from $R=1.512 \mathrm{~m}$ and the Poincare plot is shown in Fig. 3.

\section{3D MHD EQUILIBRIUM}

We compute 3D equilibria with imposed nested magnetic flux surfaces using the VMEC code. ${ }^{1}$ In this work, we employ a fixed boundary version of this code. As inputs, we require the pressure profile, the toroidal plasma current profile which we choose to vanish on each flux surface, and the Fourier amplitudes of $R$ and $Z$ (the distance from the midplane) of the plasma boundary. We Fourier decompose a fit to the outermost flux surface that we have identified with the field line tracing for each configuration using an optimal spectral condensation method. ${ }^{20} \mathrm{~A}$ truncated spectrum is chosen with poloidal modes $0 \leqslant m_{v} \leqslant 10$ and toroidal modes (per period) $-7 \leqslant n_{v} \leqslant 7$. To verify the accuracy of the fit,

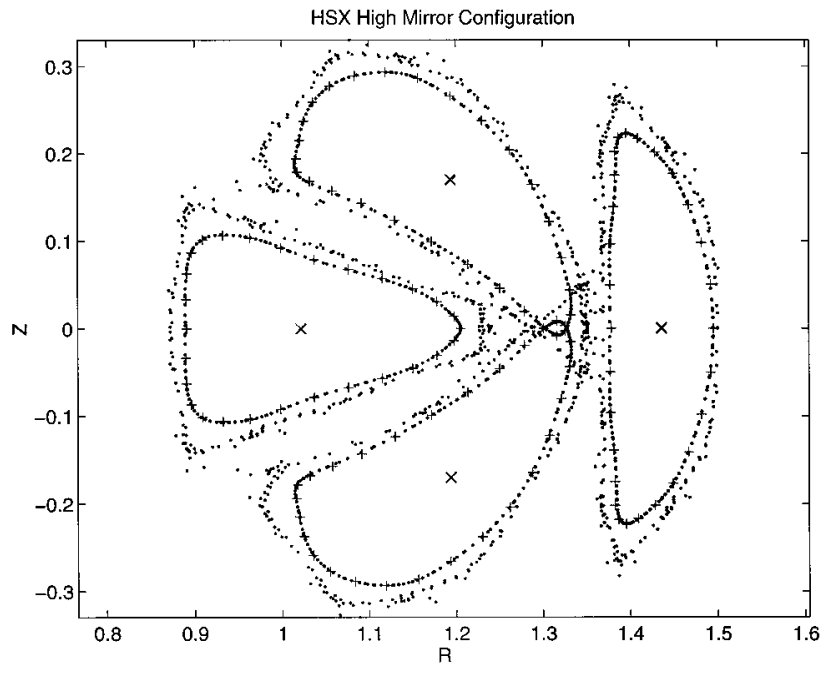

FIG. 2. Poincaré plot obtained from tracing a vacuum magnetic field line started at $R=1.495 \mathrm{~m}, Z=0$ on the elongated up-down symmetric toroidal cross section on 4 planes within one field period (inner dots) for the high magnetic mirror HSX configuration. This surface is Fourier analyzed and then reconstructed in real space to yield the + symbols. The $\times$ identifies the location of the magnetic axis in the vacuum. The outer dots constitute a Poincaré plot obtained from tracing a field line started $5 \mathrm{~mm}$ further out at $R=1.5 \mathrm{~m}$.

we reconstruct the plasma boundary for each configuration. The + symbols that depict this reconstruction appear to be superimposed almost exactly onto the curves mapped by the Poincare plots shown in the figures.

The differential volume profiles for the vacuum equilibria computed with VMEC are shown for each case considered in Fig. 4 from which we extract the magnetic well values we have quoted. The rotational transform profiles in the vacuum equilibrium state for the 3 configurations considered as well

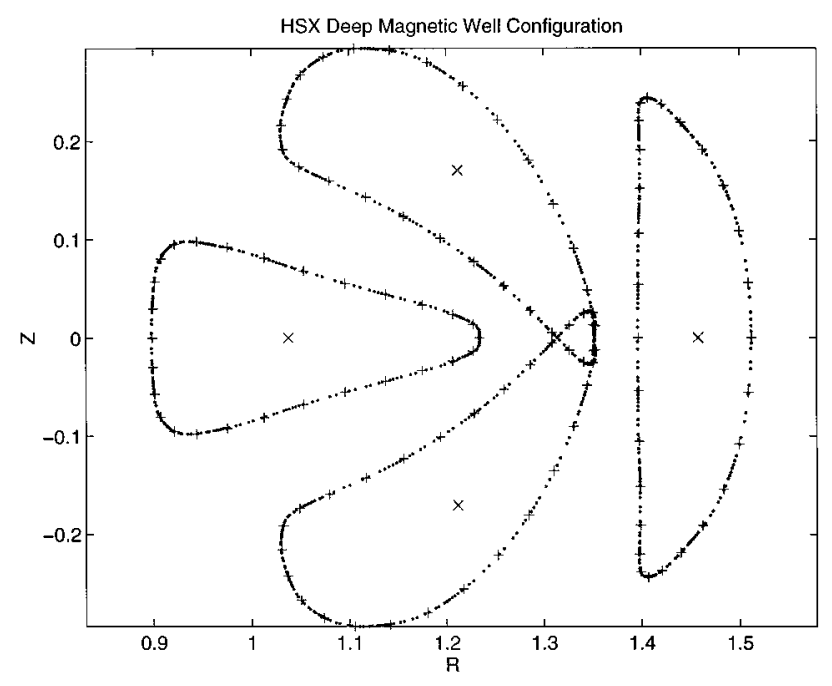

FIG. 3. Poincaré plot obtained from tracing a vacuum magnetic field line started at $R=1.512 \mathrm{~m}, Z=0$ on the elongated up-down symmetric toroidal cross section on 4 planes within one field period (dots) for the deep magnetic well HSX configuration. This surface is Fourier analyzed and then reconstructed in real space to yield the + symbols. The $\times$ identifies the location of the magnetic axis in the vacuum. 


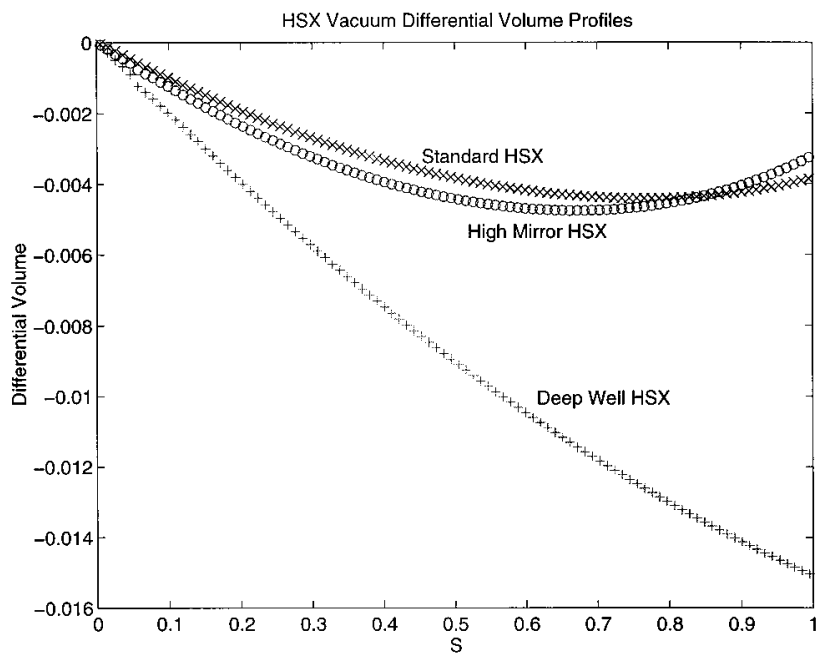

FIG. 4. The vacuum differential volume profiles computed with the VMEC code for the standard HSX configuration $(\times)$, the high magnetic mirror HSX configuration $(\bigcirc)$, and the deep magnetic well HSX configuration $(+)$. The differential volume is defined as $\left[V^{\prime}(s)-V^{\prime}(0)\right] / V^{\prime}(0)$, where $V^{\prime}=d V / d \Phi$, with $V$ the plasma volume and $\Phi$ the toroidal magnetic flux function.

as that at volume averaged $\beta=1.8 \%$ for the deep magnetic well are plotted as a function of the radial variable $s$ (which is proportional to the volume enclosed) in Fig. 5. We also describe in this figure the location of the most relevant low order rational surfaces that can affect each configuration. These are particularly important in the computation of the Mercier criterion. Thus the standard quasihelical configuration has the rotational transform per period resonant surfaces $\iota_{p}=7 / 27, \quad \iota_{p}=5 / 19$, and $\iota_{p}=4 / 15$ at radial positions

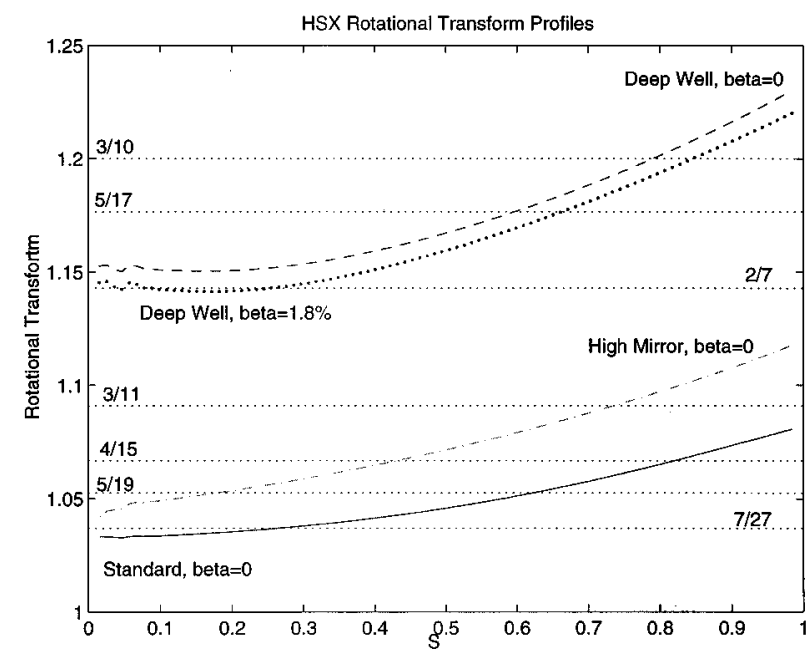

FIG. 5. The rotational transform profiles for the standard HSX configuration at $\beta=0$ (solid curve), the high mirror HSX configuration at $\beta=0$ (dotdashed curve), the deep well HSX configuration at $\beta=0$ (dashed curve), and the deep well HSX configuration at $\beta=1.8 \%$ (solid dot curve). The transform profiles for the standard and high mirror configurations near their respective $\beta$ limits do not change significantly compared with their corresponding vacuum profiles. The horizontal dotted lines identify the locations of the main low order resonant surfaces. Each line is labelled with its value of rotational transform per field period $\iota_{p}=n_{v} / m_{v}$. $s \simeq 0.27, s \simeq 0.62$, and $s \simeq 0.81$, respectively. In the high mirror case, we have $\iota_{p}=5 / 19, \iota_{p}=4 / 15$, and $\iota_{p}=3 / 11$ at $s \simeq 0.18, s \simeq 0.42$, and $s \simeq 0.74$, respectively. For the deep well configuration, we have $\iota_{p}=5 / 17$ around $s \sim 0.6$ and $\iota_{p}=3 / 10$ around $s \sim 0.8$. However, it is the resonance with $\iota_{p}=2 / 7$ which is absent in the vacuum case but which is double valued at finite $\beta$ near the center of the plasma that we can anticipate will cause problems for stability. We consider first sequences of equilibria with relatively broad pressure profiles that are obtained from a density profile given by

$$
N(s)=N(0)\left(1-s^{4}\right),
$$

and a temperature profile given by

$$
T(s)=T(0)(1-s),
$$

from which we prescribe $p=N T$. Subsequently, we analyze just for the standard quasihelical configuration a peaked pressure profile given by

$$
\begin{aligned}
p(s)= & p(0)\left[1 / 11(1-s)+5 / 11(1-s)^{3}\right. \\
& \left.+5 / 11(1-s)^{4}\right],
\end{aligned}
$$

\section{LOCAL IDEAL MHD STABILITY RESULTS}

In order to evaluate the stability of the equilibria we are investigating, it is convenient to reconstruct the equilibrium state in Boozer magnetic coordinates ${ }^{18}$ because in these coordinates the magnetic field lines are straight and the parallel current density is more effectively calculated. ${ }^{21}$ To accurately determine the coefficients that are required to evaluate ballooning stability, the spectrum of mode pairs that we have chosen to number 334 in the VMEC equilibrium coordinates $\left[\left(m_{v}=0,0 \leqslant n_{v} \leqslant 11\right) ;\left(1 \leqslant m_{v} \leqslant 14,-11 \leqslant n_{v} \leqslant 11\right)\right]$ is increased to 759 in the magnetic coordinate system (the VMEC spectrum plus the $n=12$ toroidal sideband on each side and $(15 \leqslant m \leqslant 50,2 \leqslant n \leqslant 12)$. Although formally ballooning stability is a sufficient condition to satisfy Mercier stability inasmuch as the Mercier criterion can be derived from an asymptotic analysis of the ballooning equation, in practice we limit the domain of the numerical calculations to encompass only 15 poloidal transits of the field line investigated. This is insufficient to resolve the extended structures of Mercier-like modes. The Mercier stability is much more efficiently calculated from a direct evaluation of the criterion. We examine the local ideal MHD stability of each configuration with the broad pressure profile described in the previous section. For the standard configuration, we consider cases with volume averaged $\beta$ of $0.4 \%, 0.5 \%, 0.6 \%$ and $0.7 \%$. The Mercier criterion as a function of $s$ is shown in Fig. 6. We see that the case is marginally stable at $\beta=0.4 \%$. The profiles are smooth except for spikes at three points. These spikes align closely with the location of the resonant surfaces with values of $\iota_{p}=7 / 27,5 / 19$, and 4/15 that were described in Fig. 5. The Mercier criterion is driven locally unstable by the singular nature of the parallel current density on the resonant surfaces. The parallel current density is calculated after invoking charge conservation $\boldsymbol{\nabla} \cdot j=0$ and equilibrium force balance to obtain the magnetic differential equation 


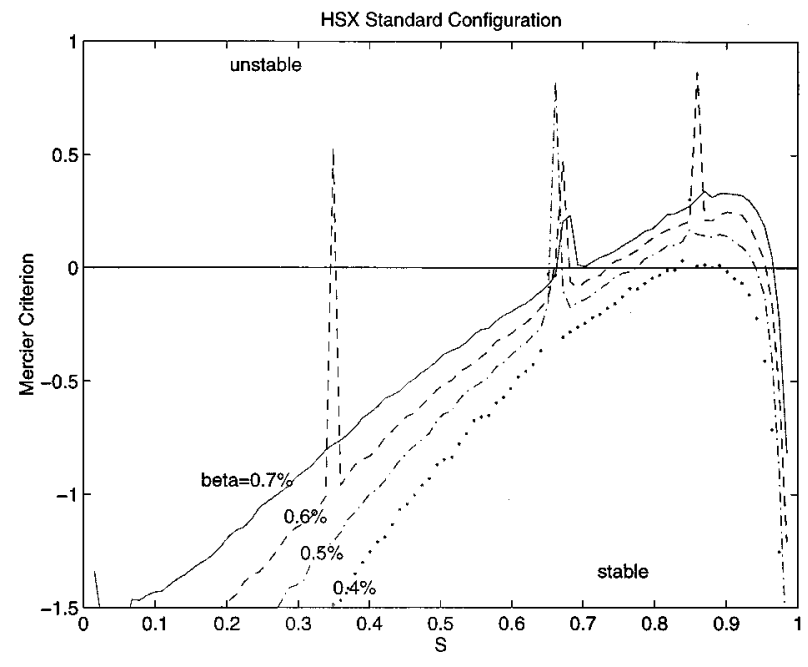

FIG. 6. The Mercier criterion with broad pressure profile for the standard HSX configuration as a function of the radial variable $s$. The solid dot curve corresponds to $\beta=0.4 \%$, the dot-dashed curve corresponds to $\beta=0.5 \%$, the dashed curve corresponds to $\beta=0.6 \%$, and the solid curve corresponds to $\beta=0.7 \%$.

$$
\sqrt{g} \mathbf{B} \cdot \nabla\left(\frac{\mathbf{j} \cdot \mathbf{B}}{B^{2}}\right)=\frac{p^{\prime}(s)}{\sqrt{g} B^{2}}\left[I(s) \frac{\partial \sqrt{g}}{\partial \theta}+J(s) \frac{\partial \sqrt{g}}{\partial \phi}\right],
$$

where $I(s)$ and $J(s)$ are the poloidal and toroidal current fluxes, respectively, $\theta$ and $\phi$ are the poloidal and toroidal angles in the Boozer magnetic coordinate system, respectively, $\mathbf{B}$ is the magnetic field, and $\sqrt{g}$ is the Jacobian. The right hand side of Eq. (4) represents the Pfirsch-Schlüter currents and they constitute the driving mechanism for the parallel current density sheets on the rational surfaces. The height of the spike is directly linked to the proximity of the mesh point (where the relevant equilibrium quantities are calculated) with respect to the exact location of the corresponding resonant surface. The calculations here are performed with 96 radial intervals. In general, when the discontinuities that are observed are localized to a single interval in 96, the structure is smaller than the typical Larmor radius of a thermal ion. The MHD model is not valid for length scales of such small magnitude and therefore singular spikes should not necessarily indicate instability. Furthermore, when the Mercier unstable spikes involve more than a couple of grid points, we believe this to constitute an indication of small island formation that smears the pressure gradient and thus regularizes the parallel current discontinuity. This proposed resolution of the problem is beyond the scope of the calculations we perform with the VMEC code which constrains the magnetic surfaces to be nested. When the unstable Mercier spikes start to coalesce at high $\beta$, we could anticipate a corresponding merging of island structures that result in the stochastization of the equilibrium field lines. The ballooning stability analysis of the sequence of equilibria reveals that the $\beta$ limit due to this type of mode approaches $0.7 \%$ as shown in Fig. 7.

The local ideal MHD stability properties of the high magnetic mirror configuration are quite similar to those of the standard configuration. Thus, both the Mercier criterion

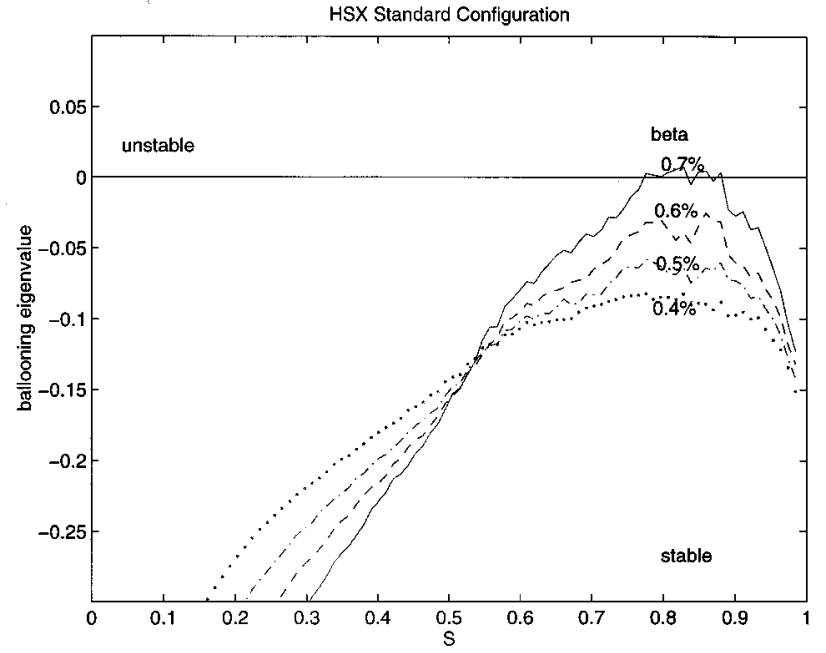

FIG. 7. The ballooning eigenvalue with broad pressure profile for the standard HSX configuration as a function of the radial variable $s$. The solid dot curve corresponds to $\beta=0.4 \%$, the dot-dashed curve corresponds to $\beta=0.5 \%$, the dashed curve corresponds to $\beta=0.6 \%$, and the solid curve corresponds to $\beta=0.7 \%$.

and the ballooning modes are destabilized near the plasma edge with the broad pressure profile considered. The edge destabilization of the Mercier criterion in these two configurations at relatively low values of $\beta$ is caused by the small local magnetic hill that forms in the outer volume of the plasma (see Fig. 4). It persists, as we shall see, even with a peaked pressure profile, albeit at somewhat higher $\beta$. The $\beta$ limit imposed by the Mercier criterion is about $0.3 \%$ and that imposed by the ballooning modes is about $0.6 \%$. In Fig. 8 , the Mercier criterion profiles also exhibit spikes that are driven by the parallel current density on rational surfaces with $\iota_{p}=5 / 19, \iota_{p}=4 / 15$, and $\iota_{p}=3 / 11$ that were identified in

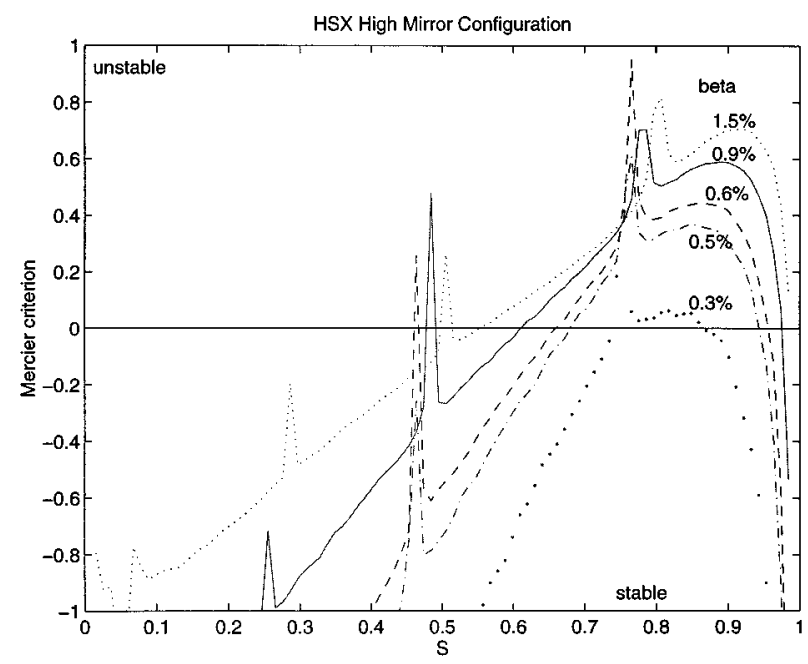

FIG. 8. The Mercier criterion with broad pressure profile for the high magnetic mirror HSX configuration as a function of the radial variable $s$. The solid dot curve corresponds to $\beta=0.3 \%$, the dot-dashed curve corresponds to $\beta=0.5 \%$, the dashed curve corresponds to $\beta=0.6 \%$, the solid curve corresponds to $\beta=0.9 \%$, and the fine dotted curve corresponds to $\beta=1.5 \%$. 


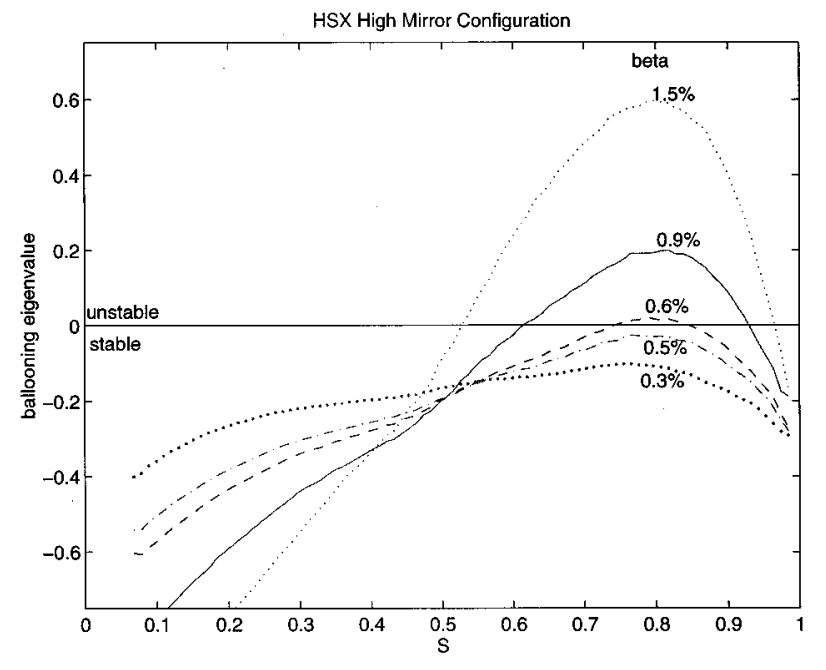

FIG. 9. The ballooning eigenvalue with broad pressure profile for the high magnetic mirror HSX configuration as a function of the radial variable $s$. The solid dot curve corresponds to $\beta=0.3 \%$, the dot-dashed curve corresponds to $\beta=0.5 \%$, the dashed curve corresponds to $\beta=0.6 \%$, the solid curve corresponds to $\beta=0.9 \%$, and the fine dotted curve corresponds to $\beta=1.5 \%$.

Fig. 5. The ballooning eigenvalue profiles for the high mirror cases considered with $\beta$ values between $0.3 \%$ and $1.5 \%$ are displayed in Fig. 9.

The $\beta$ limits imposed by local modes become significantly higher in the deep magnetic well configuration and the stability characteristics with respect to Mercier modes differ significantly compared with the two other configurations explored. The Mercier criterion profiles are shown in Fig. 10 and impose a $\beta$ limit at $1.3 \%$. But in this case, the Mercier criterion is destabilized near the center of the plasma in a region where the critical resonant surface has $\iota_{p}=2 / 7$. At

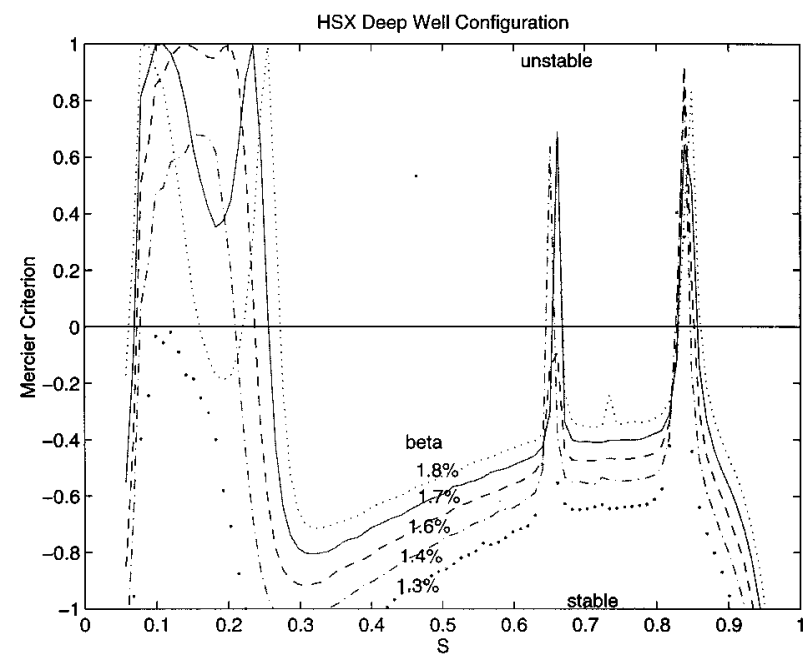

FIG. 10. The Mercier criterion with broad pressure profile for the deep magnetic well HSX configuration as a function of the radial variable $s$. The solid dot curve corresponds to $\beta=1.3 \%$, the dot-dashed curve corresponds to $\beta=1.4 \%$, the dashed curve corresponds to $\beta=1.6 \%$, the solid curve corresponds to $\beta=1.7 \%$, and the fine dotted curve corresponds to $\beta=1.8 \%$.

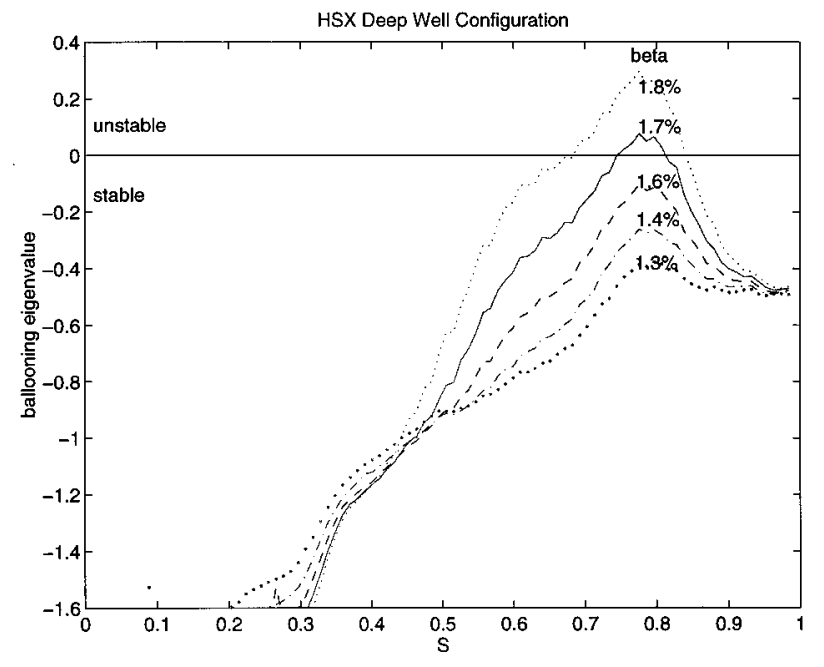

FIG. 11. The ballooning eigenvalue with broad pressure profile for the deep magnetic well HSX configuration as a function of the radial variable $s$. The solid dot curve corresponds to $\beta=1.3 \%$, the dot-dashed curve corresponds to $\beta=1.4 \%$, the dashed curve corresponds to $\beta=1.6 \%$, the solid curve corresponds to $\beta=1.7 \%$, and the fine dotted curve corresponds to $\beta=1.8 \%$.

$\beta=1.4 \%$, the resonance has not yet surfaced in the plasma but its proximity is close enough to destabilize a significant part of the central volume of the plasma. The rotational transform profile for the sequence investigated is weakly nonmonotonic and has a minimum roughly at the location where the $\iota_{p}=2 / 7$ resonance emerges in the plasma at $\beta<1.6 \%$. A further increase in $\beta$ and corresponding decrease in rotational transform causes the resonance to become double-valued in the plasma. Thus for $\beta \geq 1.6 \%$, the Mercier criterion displays a double peak, each peak aligned with the locations of the $\iota_{p}=2 / 7$ rational surfaces. As their respective positions move physically further apart at higher $\beta$, the region in between becomes stable. This appears clearly in the Mercier criterion profile for $\beta=1.8 \%$ in Fig. 10 where the $\iota_{p}=2 / 7$ resonances lie at $s \simeq 0.09$ and $s \simeq 0.27$. Unlike the other configurations explored, the magnetic well extends all the way to the plasma boundary. The edge destabilization of the Mercier criterion in this case is caused by the parallel current density spikes (driven by the Pfirsch-Schlüter currents) around the resonant surfaces with $\iota_{p}=5 / 17$ and $\iota_{p}=3 / 10$. The ballooning eigenvalues as a function of $s$ are displayed in Fig. 11 for the deep well configuration for $\beta$ values between $1.3 \%$ and $1.8 \%$. As in the other two configurations, the ballooning modes are destabilized near the plasma edge at $s \simeq 0.8$ for the type of pressure profile prescribed and yield a limit at $\beta \simeq 1.66 \%$.

The ballooning eigenvalue profiles appear to become more stable with increasing $\beta$ in the plasma core $(s \lesssim 0.5)$. This restabilization may be in effect more apparent than real because it is limited to these cases with broad pressure profile. This becomes evident when we investigate the standard configuration with the peaked pressure profile given by Eq. (3). First we show the Mercier criterion profiles in Fig. 12 and find a $\beta \simeq 0.7 \%$ limit localized near $s \simeq 0.7$ where the parallel current density about the $\iota_{p}=5 / 19$ resonance plays 


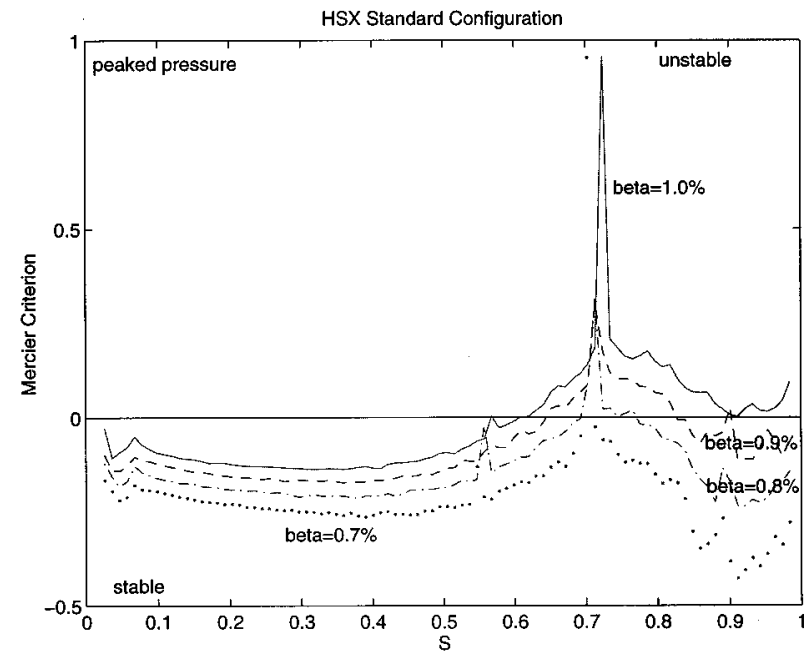

FIG. 12. The Mercier criterion with peaked pressure profile for the standard HSX configuration as a function of the radial variable $s$. The solid dot curve corresponds to $\beta=0.7 \%$, the dot-dashed curve corresponds to $\beta=0.8 \%$, the dashed curve corresponds to $\beta=0.9 \%$, and the solid curve corresponds to $\beta=1.0 \%$.

an important destabilizing effect. The behavior of the ballooning eigenvalue profiles with peaked pressure is markedly different than that with broad pressure. The core region of the plasma is destabilized by the steep central pressure gradients as seen in Fig. 13 at $\beta \geq 0.8 \%$. Therefore, the standard HSX configuration with a peaked pressure profile offers the interesting prospect that ballooning stability limits the core pressure gradient while Mercier stability limits the edge pressure gradient at a comparable value of $\beta$. The pressure profile given by Eq. (3) may thus be very close to optimal with respect to local ideal MHD stability.

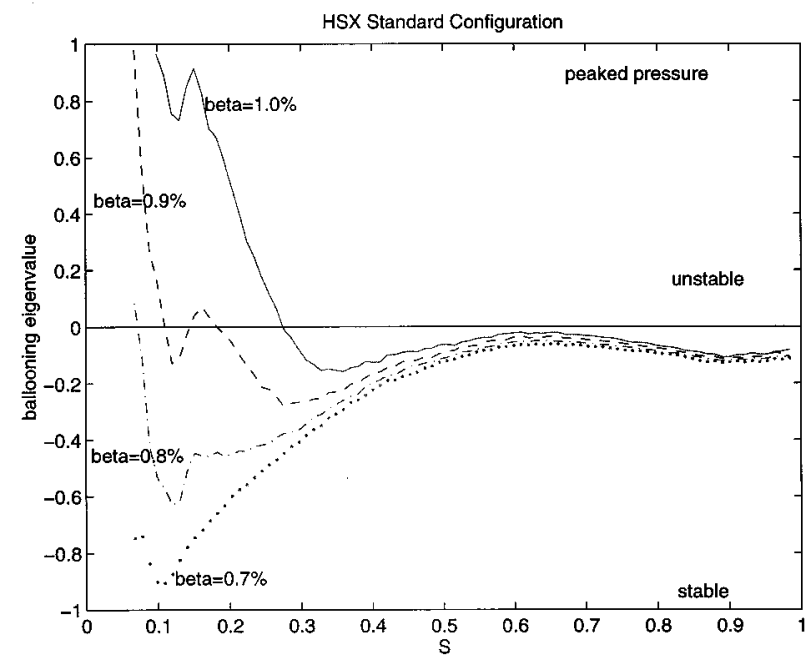

FIG. 13. The ballooning eigenvalue with peaked pressure profile for the standard HSX configuration as a function of the radial variable $s$. The solid dot curve corresponds to $\beta=0.7 \%$, the dot-dashed curve corresponds to $\beta=0.8 \%$, the dashed curve corresponds to $\beta=0.9 \%$, and the solid curve corresponds to $\beta=1.0 \%$.

\section{CONCLUSIONS}

We have investigated the local ideal MHD stability properties of three configurations that can be realized with the coil systems of the HSX device. The standard configuration is quasihelically symmetric, the high mirror configuration has neoclassical transport properties comparable to a conventional stellarator, and the deep well configuration enhances the magnetic well from $0.4 \%$ to $1.5 \%$. The plasma boundaries are determined by tracing vacuum magnetic field lines for each configuration and they are fixed as $\beta$ is increased. The toroidal plasma current is prescribed to vanish within each magnetic flux surface. In principle, a bootstrap current consistent with the pressure gradient should be prescribed. This current has two effects that could impact stability. First, it alters the rotational transform. A worse case estimate indicates a decrease in the edge transform by 0.01 corresponding to a total integrated current of less than $700 \mathrm{~A}$ for a central density of $10^{13} \mathrm{~cm}^{-3}$ and temperature of $1 \mathrm{keV}$. This change is too small to modify the results we have obtained. Second, it contributes to the parallel current density which can be a source term for instability. This effect may be more important, but can be controlled with the auxiliary coil set. A more detailed investigation of the bootstrap current impact on MHD stability is left for future work. We have employed a relatively broad pressure profile to compare predictions of 3D Mercier and 3D ballooning stability of the three configurations. We have found that the Mercier criterion imposes more restrictive $\beta$ limits than the ballooning modes, in contrast to what is found in other low shear stellarators. ${ }^{7,8}$ For this pressure profile, both Mercier and ballooning modes are destabilized near the edge of the plasma except for the deep well configuration where the parallel current density around the rational surface with $\iota_{p}=2 / 7$ destabilizes Mercier type modes near the center of the plasma. The $\beta$ limit imposed by the Mercier criterion is $1.3 \%$ and by the ballooning mode is $1.66 \%$ for the deep well configuration. The corresponding limits for the other two configurations are approximately $0.3 \%$ and $0.6 \%$, respectively. This demonstrates the sensitivity of the $\beta$ limits to the depth of the magnetic well. A near optimal pressure profile for the standard HSX configuration that is very peaked is obtained where ballooning modes near the plasma center and Mercier modes near the plasma edge combine to impose a $\beta \leqq 0.8 \%$ limit.

The flexibility of the HSX device offers the potential of very interesting comparisons of performance between the favorable transport properties expected from the standard quasihelical configuration and the large neoclassical losses that are expected with the high mirror configuration. In this paper, we have shown that the stability limits for these two configurations are roughly similar. Thus the control of the magnetic field spectrum with the auxiliary coils allows wide variations in the neoclassical transport with minimal impact on plasma stability. In addition, we have shown that the auxiliary coils can also provide access to a higher stability limit in the deep well configuration, albeit with some sacrifice in the quasihelical symmetry.

The results obtained here suggest a direction to pursue in the design of potential future advanced stellarator concepts. 
This would focus on the search for configurations that retain the quasisymmetry properties of the HSX device, especially if improved confinement is demonstrated, but that can develop a deeper vacuum magnetic well to achieve higher volume average $\beta$.

\section{ACKNOWLEDGMENTS}

We thank Dr. S.P. Hirshman for use of the VMEC equilibrium code.

J.N.T. would like to acknowledge support from the U.S. Department of Energy under Grant No. DE-FG0293ER54222. The work of W.A.C. was partially sponsored by the Fonds National Suisse de la Recherche Scientifique and by EURATOM.

${ }^{1}$ S.P. Hirshman and O. Betancourt, J. Comput. Phys. 96, 99 (1991).

${ }^{2}$ C. Mercier, Nucl. Fusion 1, 121 (1960).

${ }^{3}$ J.W. Connor, R.J. Hastie, and J.B. Taylor, Proc. R. Soc. London Ser. A 365, 1 (1979).

${ }^{4}$ D. Correa-Restrepo, Z. Naturforsch. 33a, 789 (1978).

${ }^{5}$ R.L. Dewar and A.H. Glasser, Phys. Fluids 26, 3038 (1983).

${ }^{6}$ J. Nührenberg and R. Zille, Phys. Lett. A 114, 129 (1986).

${ }^{7}$ R. Moeckli and W.A. Cooper, Nucl. Fusion 33, 1859 (1993).

${ }^{8}$ W.A. Cooper and H.J. Gardner, Nucl. Fusion 34, 729 (1994).

${ }^{9}$ See National Technical Information Service Document No. DE-96002289

[N. Dominguez and V.E. Lynch, Absence of Second Stability in ATF, Oak

Ridge National Laboratory Report ORNL/TM-13040, 1995, Nucl. Fusion (submitted)]. Copies may be ordered from the National Technical Information Service, 5285 Fort Royal Rd., Springfield, VA 22161.

${ }^{10}$ W.A. Cooper, S.P. Hirshman, and D.K. Lee, Nucl. Fusion 29, 617 (1989).

${ }^{11}$ R. Moeckli and W.A. Cooper, Phys. Plasmas 1, 793 (1994).

${ }^{12}$ W.A. Cooper, Y. Nakamura, M. Wakatani, R. Gruber, S. Merazzi, D.V. Anderson, and U. Schwenn, in Proceedings of the 19th EPS Conference on Controlled Fusion and Plasma Physics, 1992, Innsbruck, Europhysics Conference Abstracts (European Physical Society, Petit-Lancy, 1992), Vol. 16C, p. 557.

${ }^{13}$ J. Nührenberg and R. Zille, Phys. Lett. A 129, 113 (1988).

${ }^{14}$ J. Nührenberg and R. Zille, Joint Varenna-Lausanne International Workshop on Theory of Fusion Plasmas, edited by J. Vaclavic, F. Troyon, and E. Sindoni (Societa Italiana di Fisica-Editrice Compositori, Bologna, 1989), pp. 3-11.

${ }^{15}$ D.A. Garren and A.H. Boozer, Phys. Fluids B 3, 2822 (1991).

${ }^{16}$ M.Yu. Isaev, M.I. Mikhailov, and V.D. Shafranov, Plasma Phys. Rep. 20, 319 (1994).

${ }^{17}$ F.S.B. Anderson, A. Almagri, D.T. Anderson, P.G. Matthews, J.N. Talmadge, and J.L. Shohet, Trans. Fusion Tech. 27, 273 (1995)

${ }^{18}$ A.H. Boozer, Phys. Fluids 23, 904 (1980).

${ }^{19}$ P.G. Matthews, A.F. Almagri, D.T. Anderson, F.S.B. Anderson, J.L. Shohet, and J.N. Talmadge, in Proceedings, 10th International Conference on Stellarators (International Atomic Energy Agency Technical Committee Meeting), Madrid, 1995 (International Atomic Energy Agency, Vienna, 1995), p. 167; J.N. Talmadge, A.F. Almagri, F.S.B. Anderson, D.T. Anderson, P.G. Matthews, J.L. Shohet, and N. Karulin, Bull. Am. Phys. Soc. 39, 1602 (1994).

${ }^{20}$ S.P. Hirshman and H.K. Meier, Phys. Fluids 28, 1387 (1985).

${ }^{21}$ J. Nührenberg and R. Zille, in Theory of Fusion Plasmas, edited by A. Bondeson, E. Sindoni, and F. Troyon (Societa Italiana di Fisica-Editrice Compositori, Bologna, 1988), pp. 3-23. 